\title{
Pion vector form factor from lattice QCD at the physical point
}

\author{
C. Alexandrou, ${ }^{1,2}$ S. Bacchio, ${ }^{1,3}$ P. Dimopoulos, ${ }^{4,5}$ J. Finkenrath, ${ }^{6}$ R. Frezzotti, ${ }^{4}$ K. Jansen, ${ }^{7}$ B. Kostrzewa, ${ }^{8}$ \\ M. Mangin-Brinet, ${ }^{9}$ F. Sanfilippo, ${ }^{10}$ S. Simula, ${ }^{10}$ C. Urbach, ${ }^{8}$ and U. Wenger ${ }^{11}$
}

(ETM Collaboration)

\author{
${ }^{1}$ Department of Physics, University of Cyprus, P.O. Box 20537, 1678 Nicosia, Cyprus \\ ${ }^{2}$ The Cyprus Institute, 20 Kavafi Street, Nicosia 2121, Cyprus \\ ${ }^{3}$ Fakultät für Mathematik und Naturwissenschaften, Bergische Universität Wuppertal, \\ 42119 Wuppertal, Germany \\ ${ }^{4}$ Dipartimento di Fisica, Università di Roma Tor Vergata e Sezione INFN di Roma Tor Vergata, \\ Via della Ricerca Scientifica 1, I-00133 Roma, Italy \\ ${ }^{5}$ Centro Fermi-Museo Storico della Fisica e Centro Studi e Ricerche "Enrico Fermi," \\ Compendio del Viminale, Piazza del Viminale 1, 00184 Roma, Italy \\ ${ }^{6}$ Computation-based Science and Technology Research Center, The Cyprus Institute, \\ P.O. Box 27456, 1645 Nicosia, Cyprus \\ ${ }^{7}$ John von Neumann Institute for Computing (NIC), DESY, Platanenallee 6, 15738 Zeuthen, Germany \\ ${ }^{8}$ HISKP and BCTP, Rheinische Friedrich-Wilhelms Universität Bonn, 53115 Bonn, Germany \\ ${ }^{9}$ Theory Group, Lab. de Physique Subatomique et de Cosmologie, 38026 Grenoble, France \\ ${ }^{10}$ Istituto Nazionale di Fisica Nucleare, Sezione di Roma Tre, Via della Vasca Navale 84, \\ I-00146 Rome, Italy \\ ${ }^{11}$ Albert Einstein Center for Fundamental Physics, University of Bern, 3012 Bern, Switzerland
}

(Received 3 November 2017; published 29 January 2018)

\begin{abstract}
We present an investigation of the electromagnetic pion form factor, $F_{\pi}\left(Q^{2}\right)$, at small values of the fourmomentum transfer $Q^{2}\left(\lesssim 0.25 \mathrm{GeV}^{2}\right)$, based on the gauge configurations generated by the European Twisted Mass Collaboration with $N_{f}=2$ twisted-mass quarks at maximal twist including a clover term. Momentum is injected using nonperiodic boundary conditions and the calculations are carried out at a fixed lattice spacing ( $a \simeq 0.09 \mathrm{fm}$ ) and with pion masses equal to its physical value, $240 \mathrm{MeV}$ and $340 \mathrm{MeV}$. Our data are successfully analyzed using chiral perturbation theory at next-to-leading order in the light-quark mass. For each pion mass two different lattice volumes are used to take care of finite size effects. Our final result for the squared charge radius is $\left\langle r^{2}\right\rangle_{\pi}=0.443(29) \mathrm{fm}^{2}$, where the error includes several sources of systematic errors except the uncertainty related to discretization effects. The corresponding value of the $\mathrm{SU}(2)$ chiral low-energy constant $\bar{\ell}_{6}$ is equal to $\bar{\ell}_{6}=16.2(1.0)$.
\end{abstract}

DOI: 10.1103/PhysRevD.97.014508

\section{INTRODUCTION}

The investigation of the physical properties of the pion, which is the lightest bound state in quantum chromodynamics (QCD), can provide crucial information on the way low-energy dynamics is governed by the quark and gluon degrees of freedom. In this respect for spacelike values of the squared four-momentum transfer, $Q^{2} \equiv$ $-q^{2} \geq 0$, the electromagnetic (e.m.) form factor of the

Published by the American Physical Society under the terms of the Creative Commons Attribution 4.0 International license. Further distribution of this work must maintain attribution to the author(s) and the published article's title, journal citation, and DOI. Funded by SCOAP ${ }^{3}$. pion, $F_{\pi}\left(Q^{2}\right)$, parametrizes how the pion deviates from a point particle when probed electromagnetically, thus giving insight on the distribution of its charged constituents. At momentum transfer below the scale of chiral symmetry breaking $\left(Q^{2} \lesssim 1 \mathrm{GeV}^{2}\right)$ the pion form factor represents therefore an important test of nonperturbative QCD.

It is well known that for $Q^{2} \lesssim 0.5-1 \mathrm{GeV}^{2}$ the experimental data on the pion form factor [1-3] can be reproduced qualitatively by a simple monopole ansatz inspired by the vector-meson dominance (VMD) model with the contribution from the lightest vector meson $\left(M_{\rho} \simeq 0.77 \mathrm{GeV}\right)$ only. This is not too surprising in view of the fact that in the timelike region the pion form factor is dominated by the $\rho$-meson resonance. 
An interesting issue is the quark mass dependence of the pion form factor that can be addressed by SU(2) chiral perturbation theory (ChPT) known at both next-to-leading (NLO) [4] and next-to-next-to-leading (NNLO) [5] orders. The determination of $F_{\pi}\left(Q^{2}\right)$ from lattice QCD simulations provides therefore an excellent opportunity for the study of chiral logarithms. The latter are particularly important in the case of the squared pion charge radius $r_{\pi}^{2}$, i.e., the slope of pion form factor at $Q^{2}=0$. This means also that a controlled extrapolation to the physical point is a delicate endeavor, such that one would ideally like to perform the computation directly at the physical pion mass.

Initial studies of the pion form factor using lattice QCD in the quenched approximation date back to the late 1980s [6,7] giving strong support to the vector-meson dominance hypothesis at low $Q^{2}$. Studies of $F_{\pi}\left(Q^{2}\right)$ employing unquenched simulations have been carried out in Refs. [8-15] using pion masses above the physical one and adopting ChPT as a guide to extrapolate the lattice results down to the physical pion point. Recently a computation of $F_{\pi}\left(Q^{2}\right)$ at the physical pion mass has been provided in Ref. [16].

In this work we present a determination of the pion form factor using the gauge configurations generated in Ref. [17] by the European Twisted Mass Collaboration (ETMC) with $N_{f}=2$ twisted-mass quarks at maximal twist, which guarantees the automatic $\mathcal{O}(a)$ improvement [18]. The calculations are carried out at a fixed lattice spacing $(a \simeq 0.09 \mathrm{fm})$ and with pion masses equal to its physical value, $240 \mathrm{MeV}$ and $340 \mathrm{MeV}$. Momentum is injected using nonperiodic boundary conditions in order to get values of $Q^{2}$ between $\simeq 0.01 \mathrm{GeV}^{2}$ and $\simeq 0.25 \mathrm{GeV}^{2}$. It will be shown that our data can be successfully analyzed using $\mathrm{SU}(2) \mathrm{ChPT}$ at NLO without the need of the scale setting.

Our final result for the squared pion charge radius is

$$
\left\langle r^{2}\right\rangle_{\pi}=0.443(29) \mathrm{fm}^{2},
$$

where the error includes several sources of systematic errors except the uncertainty related to discretization effects. The corresponding value of the NLO SU(2) lowenergy constant (LEC) $\bar{\ell}_{6}$ is equal to

$$
\bar{\ell}_{6}=16.2(1.0) \text {. }
$$

Our result (1) is obtained at a fixed value of the lattice spacing and therefore the continuum limit still needs to be evaluated. We note that discretization effects in our calculations of the pion form factor start at order $\mathcal{O}\left(a^{2}\right)$ (see Sec. III) and that our finding (1) is consistent with the experimental value $\left\langle r^{2}\right\rangle_{\pi}^{\exp }=0.452(11) \mathrm{fm}^{2}$ from PDG [19]. This suggests that the impact of discretization effects on our result (1) could be small with respect to the other sources of uncertainties.

The plan of the paper is as follows. In Sec. II we describe the lattice setup adopted in this work, while the procedures adopted to extract the pion form factor from appropriate ratios of three- and two-point correlators are discussed in Sec. III. The lattice data for the pion form factor $F_{\pi}\left(Q^{2}\right)$ are presented in Sec. IV and in the Appendix, while our fitting procedures based on ChPT are described in Sec. V. The results of the extrapolations to the physical point and to the infinite lattice volume are collected in Sec. VI. Our conclusions are summarized in Sec. VII.

\section{LATTICE ACTION}

The results presented in this paper are based on the gauge configurations generated in Ref. [17] by the ETMC with Wilson clover twisted-mass quark action at maximal twist [20], employing the Iwasaki gauge action [21]. The measurements are performed on $N_{f}=2$ ensembles with pion mass at its physical value, $240 \mathrm{MeV}$ and $340 \mathrm{MeV}$, respectively. The lattice spacing is $a \simeq 0.0914(15) \mathrm{fm}$ for all the ensembles [17]. In Table I we list the ensembles with the relevant input parameters, the lattice volume and the number of configurations used. More details about the ensembles are presented in Ref. [17].

Both the sea and valence quarks are described by the Wilson clover twisted-mass action. The Dirac operator for the light-quark doublet consists of the Wilson twisted-mass Dirac operator [20] combined with the clover term, namely, in the so-called physical basis

$$
D_{\ell}=D-i \gamma_{5} \tau_{3}\left[W_{\mathrm{cr}}+\frac{i}{4} c_{\mathrm{sw}} \sigma^{\mu \nu} \mathcal{F}^{\mu \nu}\right]+\mu_{\ell}
$$

TABLE I. The gauge ensembles used in this study. The labeling of the ensembles follows the notations in Ref. [17]. In addition to the relevant input parameters we give the lattice volume $(L / a)^{3} \times T / a$, the number of evaluated configurations $N_{\text {conf }}$, the pion mass $M_{\pi}$ in lattice units (with its statistical error) and the value of $M_{\pi} L$.

\begin{tabular}{lccccccc}
\hline \hline Ensemble & $\beta$ & $c_{\mathrm{sw}}$ & $a \mu_{\ell}$ & $(L / a)^{3} \times T / a$ & $N_{\text {conf }}$ & $a M_{\pi}$ & $M_{\pi} L$ \\
\hline$c A 2.09 .64$ & 2.10 & 1.57551 & 0.009 & $64^{3} \times 128$ & 360 & $0.06204(6)$ & 4.0 \\
$c A 2.09 .48$ & 2.10 & 1.57551 & 0.009 & $48^{3} \times 96$ & 615 & $0.06216(8)$ & 3.0 \\
$c A 2.30 .48$ & 2.10 & 1.57551 & 0.030 & $48^{3} \times 96$ & 345 & $0.11198(9)$ & 5.4 \\
$c A 2.30 .24$ & 2.10 & 1.57551 & 0.030 & $24^{3} \times 48$ & 300 & $0.11567(85)$ & 2.8 \\
$c A 2.60 .32$ & 2.10 & 1.57551 & 0.060 & $32^{3} \times 64$ & 330 & $0.15773(25)$ & 5.0 \\
$c A 2.60 .24$ & 2.10 & 1.57551 & 0.060 & $24^{3} \times 48$ & 270 & $0.15861(83)$ & 3.8 \\
\hline \hline
\end{tabular}


where $D=\gamma_{\mu}\left(\nabla_{\mu}^{*}+\nabla_{\mu}\right) / 2, \nabla_{\mu}$ and $\nabla_{\mu}^{*}$ are the forward and backward lattice covariant derivatives, and $W_{\text {cr }}=$ $-(a / 2) \nabla_{\mu}^{*} \nabla_{\mu}+m_{\mathrm{cr}}$ with $m_{\mathrm{cr}}$ being the critical mass. Moreover, $\mu_{\ell}$ is the average up/down (twisted) quark mass, $a$ is the lattice spacing, and $r=1$ the Wilson parameter. The operator $D_{\ell}$ acts on a flavor doublet spinor $\psi=$ $(u, d)^{T}$. Finally, $c_{\mathrm{sw}}$ is the so-called Sheikoleslami-Wohlert improvement coefficient [22] multiplying the clover term. In our case the latter is not used for $\mathcal{O}(a)$ improvement but serves to significantly reduce the effects of isospin breaking [17].

The critical mass has been determined as described in Refs. $[23,24]$. This guarantees that all physical observables can be extracted from lattice estimators that are $\mathrm{O}(\mathrm{a})$ improved by symmetry [18], which is one of the main advantages of the Wilson twisted-mass formulation of lattice QCD.

\section{THE PION FORM FACTOR}

The pion form factor can be computed from the matrix elements of the e.m. vector current

$$
V_{\mu}(x)=\frac{2}{3} \bar{u}(x) \gamma_{\mu} u(x)-\frac{1}{3} \bar{d}(x) \gamma_{\mu} d(x)
$$

between pion states, yielding

$$
\left\langle\pi^{+}\left(\overrightarrow{p^{\prime}}\right)\left|V_{\mu}(0)\right| \pi^{+}(\vec{p})\right\rangle=\left(p_{\mu}^{\prime}+p_{\mu}\right) F_{\pi}\left(Q^{2}\right),
$$

where $q_{\mu}=\left(p_{\mu}-p_{\mu}^{\prime}\right)$ is the four-momentum transfer and $Q^{2} \equiv-q^{2}$. As detailed in Ref. [9], up to discretization effects of order $\mathcal{O}\left(a^{2}\right)$ it is enough to compute in Eq. (5) only the connected insertion of the single flavor current $\bar{u}(x) \gamma_{\mu} u(x)$ with unitary charge.

Working in Euclidean space-time, we can access the region of spacelike momentum transfer, $Q^{2}=-q^{2}>0$, by evaluating ratios of pion two-point and three-point functions with the vector current insertion. To inject arbitrary momenta, we make use of nonperiodic boundary conditions (BCs) [25-27] on the quark fields. Enforcing $\psi\left(x+\vec{e}_{i} L\right)=e^{2 \pi i \theta_{i}} \psi(x)$ on the quark field $\psi$, changes the momentum quantization condition in finite volume to $p_{i}=\frac{2 \pi \theta_{i}}{L}+\frac{2 \pi n_{i}}{L}$. This is depicted in Fig. 1 for the pion threepoint function with independent values of the vector $\vec{\theta}$ for the three quark lines. Since the ETMC gauge ensembles have been produced by imposing antiperiodic BCs in time, the same conditions are applied also to the valence quarks choosing $2 \pi \theta_{0} / T=\pi / T$. Moreover, the use of different BCs in space for sea and valence quarks produces unitarity violating finite volume effects, which are however exponentially small [28-30].

For the case of twisted-mass quarks, this setup was first studied in Ref. [9] in the Breit frame $\left(\overrightarrow{p^{\prime}}=-\vec{p}\right)$, which results in a squared four-momentum transfer independent of the pion mass, viz.

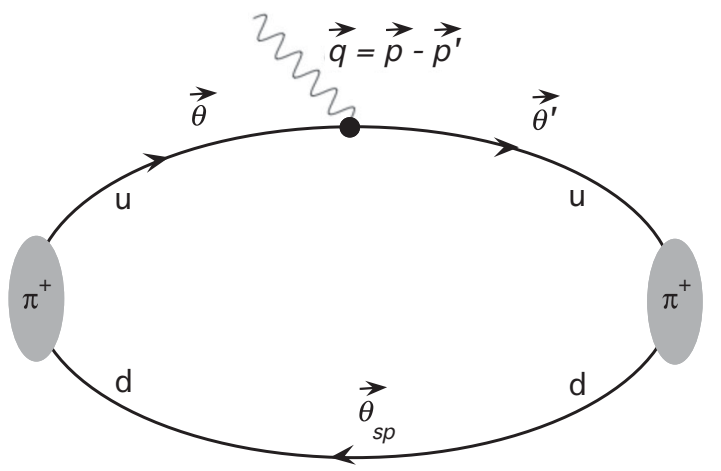

FIG. 1. Nonperiodic boundary conditions in the pion threepoint function for arbitrary quark momenta.

$$
Q^{2} \equiv-q^{2}=\left|\vec{p}-\overrightarrow{p^{\prime}}\right|^{2}-\left[E_{\pi}(\vec{p})-E_{\pi}\left(\overrightarrow{p^{\prime}}\right)\right]^{2}=4|\vec{p}|^{2}
$$

To obtain Breit frame kinematics with nonperiodic BCs, we set $\vec{\theta}^{\prime}=-\vec{\theta}$ and $\vec{\theta}_{s p}=0$ (see Fig. 1). In this work the spatial components of the vector $\vec{\theta}$ are always chosen to be equal each other, i.e., $\vec{\theta}=\{\theta, \theta, \theta\}$.

Following Ref. [9] the required correlation functions can be evaluated efficiently through the usage of the so-called one-end trick combined with spatial all-to-all propagators from stochastic time-slice sources and the sequential propagator method for the insertion (see Ref. [31] for the idea first applied to moments of pion parton distribution functions). Since the spatial matrix elements of the vector current are vanishing in the Breit frame, we have to compute the following correlation functions:

$$
\begin{aligned}
& C^{2 \mathrm{pt}}(t, \vec{p})=\sum_{\vec{x}, \vec{z}}\left\langle O_{\pi}\left(\vec{x}, t+t_{z}\right) O_{\pi}^{\dagger}\left(\vec{z}, t_{z}\right)\right\rangle e^{-i \vec{p} \cdot(\vec{x}-\vec{z})} \\
& C_{0}^{3 \mathrm{pt}}\left(t, t^{\prime}, \vec{p},-\vec{p}\right) \\
& =\sum_{\vec{x}, \vec{y}, \vec{z}}\left\langle O_{\pi}\left(\vec{y}, t^{\prime}+t_{z}\right) V_{0}\left(\vec{x}, t+t_{z}\right) O_{\pi}^{\dagger}\left(\vec{z}, t_{z}\right)\right\rangle \\
& \quad \times e^{-i \vec{p} \cdot(\vec{x}-\vec{z})-i \vec{p} \cdot(\vec{x}-\vec{y})},
\end{aligned}
$$

where $V_{0}(x)=\bar{u}(x) \gamma_{0} u(x)$ is the temporal component of the local vector current, $O_{\pi}(x)=\bar{d}(x) \gamma_{5} u(x)$ is the interpolating operator annihilating the $\pi^{+}, t$ is the time distance between the vector current insertion and the source and $t^{\prime}$ is the time distance between the sink and the source.

As it has been shown in Ref. [18], the calculation of correlation functions of globally parity invariant operators is automatically $\mathcal{O}(a)$ improved at maximal twist. Thus, for nonvanishing values of the spatial momenta the $\mathcal{O}(a)$ terms can be eliminated by appropriate averaging of the correlation functions over initial and final momenta of opposite sign. Using the invariance of our lattice formulation under an even number of space or time inversions and under 
charge conjugation as well as the $\gamma_{5}$-Hermiticity property, one gets that: (i) the correlators (6) and (7) are real, and (ii) $C^{2 \mathrm{pt}}(t, \vec{p})=C^{2 \mathrm{pt}}(t,-\vec{p})$ and $C_{0}^{3 \mathrm{pt}}\left(t, t^{\prime}, \vec{p},-\vec{p}\right)=$ $C_{0}^{3 \mathrm{pt}}\left(t, t^{\prime},-\vec{p}, \vec{p}\right)$. Thus, we have $E_{\pi}(\vec{p})=E_{\pi}(-\vec{p})$ and the discretization effects in both $C^{2 \mathrm{pt}}(t, \vec{p})$ and $C_{0}^{3 \mathrm{pt}}\left(t, t^{\prime}, \vec{p},-\vec{p}\right)$ start automatically at order $\mathcal{O}\left(a^{2}\right)$.

Taking the appropriate limits with $T$ being the time extent of the lattice, one obtains in the Breit frame

$$
\begin{gathered}
\lim _{\substack{t \rightarrow \infty \\
T \rightarrow \infty}} C^{2 \mathrm{pt}}(t, \vec{p}) \rightarrow \frac{G_{\pi}^{2}}{2 E_{\pi}(\vec{p})} e^{-E_{\pi}(\vec{p}) t} \\
\lim _{\substack{t \rightarrow \infty \\
(t \rightarrow) \infty \\
T \rightarrow \infty}} C_{0}^{3 \mathrm{pt}}\left(t, t^{\prime}, \vec{p},-\vec{p}\right) \rightarrow \frac{G_{\pi}^{2}}{2 E_{\pi}(\vec{p}) 2 E_{\pi}(\vec{p})} \\
\quad \times\left\langle\pi^{-}(\vec{p})\left|V_{0}\right| \pi^{+}(\vec{p})\right\rangle e^{-E_{\pi}(\vec{p}) t} e^{-E_{\pi}(\vec{p})\left(t^{\prime}-t\right)},
\end{gathered}
$$

where $G_{\pi}^{2}$ is the amplitude of the two-point correlation function. Since we work from now on exclusively in the Breit frame, we will drop the second momentum argument and write

$$
C_{0}^{3 \mathrm{pt}}\left(t, t^{\prime}, \vec{p},-\vec{p}\right) \equiv C_{0}^{3 \mathrm{pt}}\left(t, t^{\prime}, \vec{p}\right)
$$

Now, we can construct the ratio

$$
R\left(t, t^{\prime}, \vec{p}\right)=\frac{C_{0}^{3 \mathrm{pt}}\left(t, t^{\prime}, \vec{p}\right)}{C^{2 \mathrm{pt}}\left(t^{\prime}, \vec{p}\right)}
$$

which has the following combined limit:

$$
\lim _{\substack{t \rightarrow \infty \\ \text { and } \\ t_{T} \rightarrow \infty}} R\left(t, t^{\prime}, \vec{p}\right) \rightarrow \frac{\left\langle\pi^{+}(-\vec{p})\left|V_{0}\right| \pi^{+}(\vec{p})\right\rangle}{2 E_{\pi}(\vec{p})}=\frac{1}{Z_{V}} F_{\pi}\left(Q^{2}\right) .
$$

To extract $F_{\pi}\left(Q^{2}\right)$, we compute the renormalization constant of the vector current, $Z_{V}$, from the ratio of the twoand three-point functions at zero momentum transfer and the known normalization $F_{\pi}(0)=1$, which implies

$$
\lim _{\substack{t \rightarrow \infty \\ t \rightarrow \rightarrow \infty \\ T \rightarrow t \rightarrow \infty}} \frac{C^{2 \mathrm{pt}}(t, \overrightarrow{0})}{C_{0}^{3 \mathrm{pt}}\left(t, t^{\prime}, \overrightarrow{0}\right)} \rightarrow Z_{V}
$$

In practice, Eqs. (6) and (7) are evaluated by first generating stochastic sources $\xi_{r}^{a, \alpha}(\vec{x}, t)(r=1, \ldots, N)$ at a single (randomly chosen) time slice, that for ease of notation we conventionally put in what follows at $t_{\text {source }}=0$, namely

$$
\begin{aligned}
\lim _{N \rightarrow \infty} \frac{1}{N} \sum_{r=1}^{N} \xi_{r}^{a, \alpha}(\vec{x}, 0)^{\star} \cdot \xi_{r}^{b, \beta}(\vec{y}, 0) & =\delta_{a, b} \delta_{\alpha, \beta} \delta_{\vec{x}, \vec{y}}, \\
\lim _{N \rightarrow \infty} \frac{1}{N} \sum_{r=1}^{N} \xi_{r}^{a, \alpha}(\vec{x}, t) \cdot \xi_{r}^{b, \beta}(\vec{y}, t) & =0 .
\end{aligned}
$$

Here, $a(b)$ and $\alpha(\beta)$ are color and Dirac indices, respectively, and we remind that $t$ represents the time distance from the source. The stochastic source $\xi_{r}$ is manifestly zero for all $t \neq 0$. Setting $S_{\ell}^{\vec{\theta}} \equiv\left(D_{\ell}^{\vec{\theta}}\right)^{-1}$ and

$$
\eta_{r, \ell}^{\vec{\theta}}(\vec{x}, t)=\sum_{\vec{x}} S_{\ell}^{\vec{\theta}}\left(\vec{x}, t ; \vec{x}^{\prime}, 0\right) \cdot \xi_{r}\left(\vec{x}^{\prime}, 0\right), \quad \ell=u, d
$$

one can estimate

$\sum_{\vec{x}, a, \alpha} \eta_{r, u}^{a, \alpha, \vec{\theta}}(\vec{x}, t) \cdot\left[\eta_{r, d}^{a, \alpha, \overrightarrow{0}}(\vec{x}, t)\right]^{\star}=C^{2 \mathrm{pt}}(t, \vec{p})+$ noise

owing to $\gamma_{5}$-Hermiticity $\gamma_{5} D_{u} \gamma_{5}=D_{d}^{\dagger}$ and $\gamma_{5}^{2}=1$. In Eq. (14) the pion momentum $\vec{p}$ is given by $\vec{p}=$ $2 \pi \vec{\theta} / L$. At fixed values of $t^{\prime}$ (the time distance between the sink and the source) the so-called sequential propagator is computed as

$\varphi_{r, \ell, \ell^{\prime}}^{\vec{\theta}, \vec{\theta}^{\prime}}\left(\vec{x}, t ; t^{\prime}\right)=\sum_{\vec{x}^{\prime}} S_{\ell^{\prime}}^{\vec{\theta}^{\prime}}\left(\vec{x}, t ; \vec{x}^{\prime}, t^{\prime}\right) \cdot\left[\gamma_{5} \eta_{r, \ell}^{\vec{\theta}}\left(\vec{x}^{\prime}, t^{\prime}\right)\right]$.

Then, one estimates the three-point function in the Breit frame kinematics from

$$
\begin{aligned}
& \sum_{\vec{x}, a, \alpha, \alpha^{\prime}} \eta_{r, u}^{a, \alpha, \vec{\theta}}(\vec{x}, t) \cdot\left[\varphi_{r, d, u}^{a, \alpha^{\prime}, \overrightarrow{0},-\vec{\theta}}\left(\vec{x}, t ; t^{\prime}\right)\right]^{\star}\left(\gamma_{5} \gamma_{0}\right)_{\alpha^{\prime} \alpha} \\
& \propto C_{0}^{3 \mathrm{pt}}\left(t, t^{\prime}, \vec{p}\right)+\text { noise }
\end{aligned}
$$

We determine $F_{\pi}\left(Q^{2}\right)$ from the ratio defined in Eq. (10) in two different ways: for the first one we compute the double ratio

$$
M_{n}\left(t, t^{\prime}, \vec{p}\right) \equiv \frac{R\left(t, t^{\prime}, \vec{p}\right)}{R\left(t, t^{\prime}, \overrightarrow{0}\right)}
$$

and we extract the pion form factor from its large time distance behavior

$$
F_{\pi}\left(Q^{2}\right)=\lim _{\substack{t \rightarrow \infty \\\left(t^{\prime} \rightarrow t\right) \\ T \rightarrow \infty}} M_{n}\left(t, t^{\prime}, \vec{p}\right)
$$

We denote this estimate as the numerical one. The second estimate consists in replacing the pseudoscalar two-point function by its analytical expression, i.e., we fit 


$$
C^{2 \mathrm{pt}}(t, \vec{p})=\frac{G_{\pi}^{2}}{2 E_{\pi}(\vec{p})}\left[e^{-E_{\pi}(\vec{p}) t}+e^{-E_{\pi}(\vec{p})(T-t)}\right]
$$

to the data for the two-point function at large Euclidean times to determine the amplitude $G_{\pi}$ and the energy $E(\vec{p})$. Next we define

$$
R_{a}\left(t, t^{\prime}, \vec{p}\right)=\frac{2 E_{\pi}(\vec{p})}{G_{\pi}^{2}} \frac{C_{0}^{3 \mathrm{pt}}\left(t, t^{\prime}, \vec{p}\right)}{e^{-E_{\pi}(\vec{p}) t}+e^{-E_{\pi}(\vec{p})(T-t)}},
$$

where we replace the data for the two-point function by its analytical expression using the best fit parameters. Then we calculate the double ratio

$$
M_{a}\left(t, t^{\prime}, \vec{p}\right) \equiv \frac{R_{a}\left(t, t^{\prime}, \vec{p}\right)}{R_{a}\left(t, t^{\prime}, \overrightarrow{0}\right)},
$$

from which the pion form factor can be obtained as

$$
F_{\pi}\left(Q^{2}\right)=\lim _{\substack{t \rightarrow \infty \\\left(t^{\prime} \rightarrow t\right) \\ T \rightarrow \infty}} M_{a}\left(t, t^{\prime}, \vec{p}\right)
$$

The analytical estimate (21) may have the advantage of being less noisy than the numerical one (18), because the data for the two-point function at large $t$ can be noisy, in particular for the largest values of $|\vec{p}|$.

A further improvement is to replace in Eq. (19) the pion energy $E_{\pi}(\vec{p})$, extracted from the two-point correlator $C^{2 \mathrm{pt}}(t, \vec{p})$, with the corresponding value from the dispersion relation

$$
E_{\pi}^{\mathrm{disp}}\left(\vec{p}=\frac{2 \pi \vec{\theta}}{L}\right)=\sqrt{M_{\pi}^{2}(L)+\left(\frac{2 \pi \vec{\theta}}{L}\right)^{2}},
$$

where $M_{\pi}(L)$ is pion mass extracted from the two-point correlator at rest. Indeed, in Fig. 2 we show the measured energy levels $\left(a E_{\pi}\right)^{2}$ in lattice units as a function of the squared momentum $|a \vec{p}|^{2}$ for the two ensembles cA2.30.24 and c.A2.30.48 with $L / a=24$ and 48 , respectively. The data are described reasonably by the dispersion relation (22), indicated by the solid lines, up to the largest values of momenta $|a \vec{p}|^{2} \sim 0.01$ adopted in this work. This suggests that the main bulk of finite volume effects (FVEs) on the pion energy $E_{\pi}(\vec{p})$ originates from those of the pion mass $M_{\pi}(L)$.

However, the use of nonperiodic BCs is expected to produce further FVEs in the dispersion relation (22). Such corrections have been investigated in Ref. [32] using partially quenched ChPT at NLO, finding that the pion momentum $\vec{p}=2 \pi \vec{\theta} / L$ acquires an additive correction term $2 \pi \vec{K} / L$, namely

$$
E_{\pi}(\vec{p})=\sqrt{M_{\pi}^{2}(L)+\left(\frac{2 \pi \vec{K}}{L}+\frac{2 \pi \vec{\theta}}{L}\right)^{2}},
$$

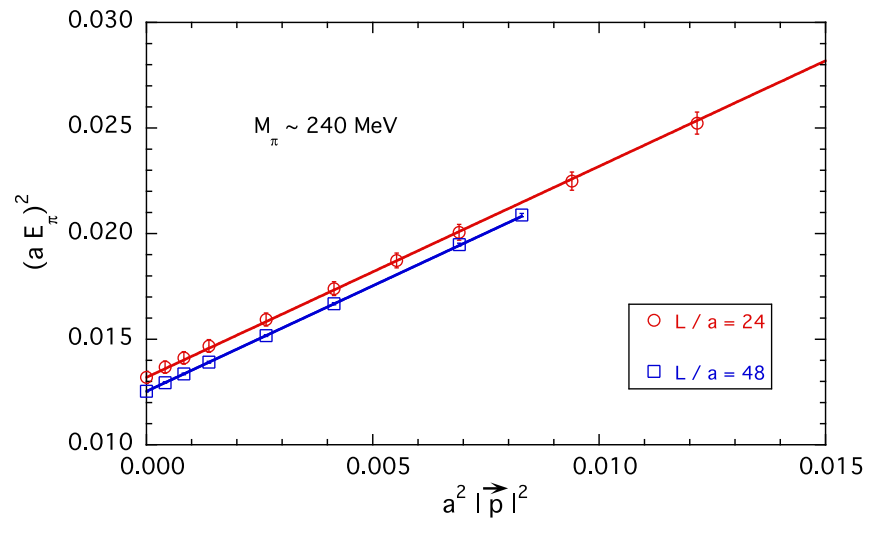

FIG. 2. Pion dispersion relation for the gauge ensembles cA2.30.24 and cA2.30.48 at a pion mass $M_{\pi} \simeq 240 \mathrm{MeV}$ with $L / a=24$ and 48, respectively. The solid lines represent the continuum dispersion relation (22) for the two gauge ensembles.

where the components of the vector $\vec{K}$ are given by

$$
\begin{aligned}
K_{i}= & -\frac{1}{2 \pi^{3 / 2}\left(f_{\pi} L\right)^{2}} \int_{0}^{\infty} d \tau \frac{1}{\sqrt{\tau}} e^{-\tau\left(\frac{M_{\pi} L}{2 \pi}\right)^{2}} \bar{\Theta}\left(\tau, \theta_{i}\right) \\
& \times \prod_{j \neq i, j=1}^{3} \Theta\left(\tau, \theta_{j}\right)
\end{aligned}
$$

with $\Theta(\tau, \theta) \equiv \sum_{n=-\infty}^{\infty} e^{-\tau(n+\theta)^{2}}$ and $\bar{\Theta}(\tau, \theta) \equiv \sum_{n=-\infty}^{\infty}$ $(n+\theta) e^{-\tau(n+\theta)^{2}}$ being the elliptic Jacobi function and its derivative.

For a better visualization of the effects of the additive correction (24) we consider the dimensionless quantity $c^{2}$, defined as

$$
c^{2} \equiv \frac{E_{\pi}^{2}(\vec{p})-M_{\pi}^{2}(L)}{|\vec{p}|^{2}}=\frac{|\vec{K}+\vec{\theta}|^{2}}{|\vec{\theta}|^{2}},
$$

which in absence of FVEs on the momentum should be equal to unity. In Fig. 3 the values of $c^{2}$ corresponding to the energy $E_{\pi}(\vec{p})$ and the mass $M_{\pi}(L)$, extracted from the appropriate two-point correlators, are shown for various values of $|a \vec{p}|^{2}$ for the gauge ensemble cA2.09.48 and cA2.30.24. It can be seen that $c^{2}$ deviates from unity and its momentum dependence is consistent with the NLO ChPT prediction corresponding to Eqs. (23) and (24) at the largest values of $|a \vec{p}|^{2}$, while the trend of the data is not reproduced at small values of the momentum, even if the present precision does not allow us to draw definite conclusions. This issue certainly deserves further investigations, which are however outside the scope of the present work.

In order to minimize excited state effects, the source-sink separation is fixed to $t^{\prime}=T / 2$. On each gauge configuration, multiple source time slices are chosen randomly across the whole time extent, which has been shown to 

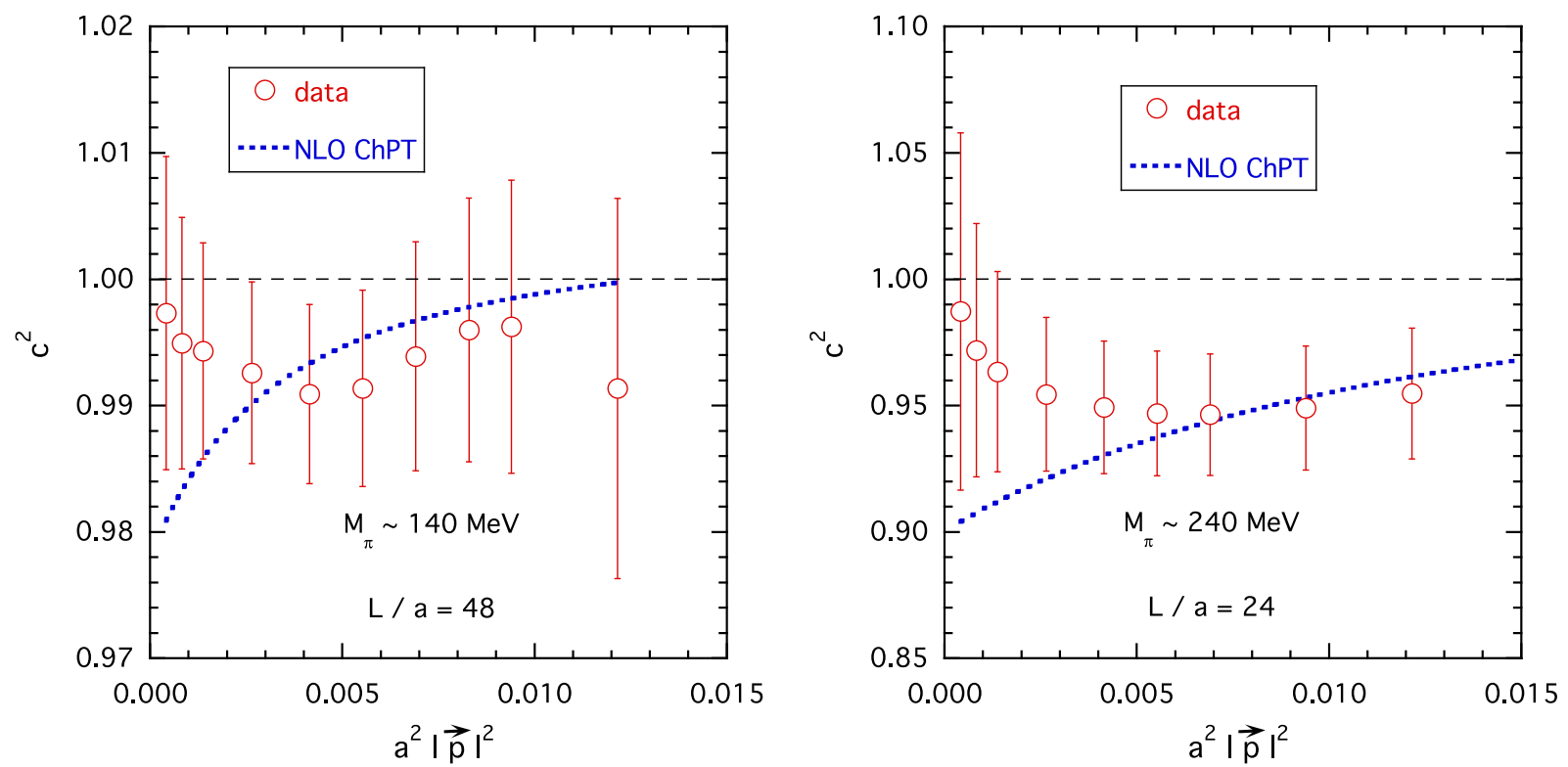

FIG. 3. The quantity $c^{2}=\left(E_{\pi}^{2}(\vec{p})-M_{\pi}^{2}(L)\right) /|\vec{p}|^{2}$ versus $a^{2}|\vec{p}|^{2}$ for the gauge ensembles cA2.09.48 (left panel) and cA2.30.24 (right panel). The dashed lines are the predictions of NLO ChPT [32] obtained from Eqs. (23) and (24). Note the different range of values for $c^{2}$ in the left and right panels.

decorrelate measurements from different gauge configurations. The statistical analysis is performed using the blocked bootstrap method.

Since $M(t, T / 2, \vec{\theta})=M(T-t, T / 2, \vec{\theta})$, we perform the averaging of forward and backward three-point correlation functions

$$
\bar{M}(t, T / 2, \vec{\theta})=\frac{1}{2}[M(t, T / 2, \vec{\theta})+M(T-t, T / 2, \vec{\theta})] .
$$

The vector form factor $F_{\pi}\left(Q^{2}\right)$ can then be extracted from the ratio $\bar{M}(t, T / 2, \vec{\theta})$ for values of $t$ in the range $\left[t_{\min }, T / 2-t_{\min }\right]$, where $t_{\min }$ is the time distance at which excited states have decayed sufficiently from both the source and the sink. The ratio $\bar{M}(t, T / 2, \vec{\theta})$ is also symmetric with respect to $t=T / 4$. The quality of the plateaus is illustrated in Fig. 4 for a few selected values of $Q^{2}$ in the case of the gauge ensemble cA2.09.64.

Before closing this section, we address briefly the estimate of the renormalization constant of the vector current, $Z_{V}$, which can be obtained form the plateau of the ratio (11). We remind that the latter one involves twoand three-point correlation functions with pion at rest and corresponds to fix the absolute normalization of the pion form factor, $F_{\pi}\left(Q^{2}=0\right)=1$. The data for the ratio (11) exhibit nice plateaus in an extended time region $(t / a \gtrsim 5)$ and allow us to extract $Z_{V}$ with a very high statistical precision $(\approx 0.01 \%)$. The resulting values of $Z_{V}$ do depend upon the quark mass as a pure discretization effect (see also Ref. [9]). The extrapolation to the chiral limit provides therefore the value of the renormalization constant $Z_{V}$,

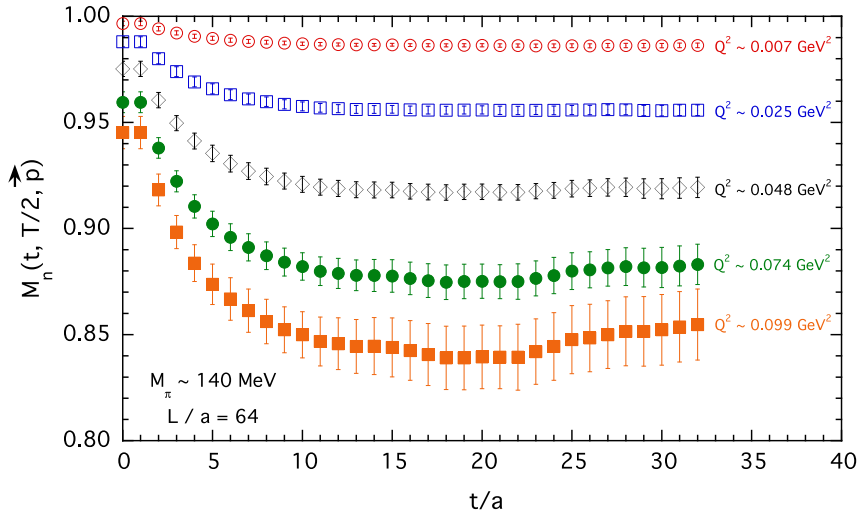

FIG. 4. The ratio $M_{n}(t, T / 2, \vec{p})$ [see Eq. (17) for $t^{\prime}=T / 2$ ] in the case of the ensemble cA2.09.64 (i.e., $T / 2=64 a$ ) for few selected values of the squared four-momentum transfer $Q^{2}$. The values of the pion form factor $F_{\pi}\left(Q^{2}\right)$ are determined from the plateaus corresponding to the time range $\left[t_{\min }, T / 2-t_{\min }\right]$ with $t_{\min }=12 a$. Since the ratio $M_{n}(t, T / 2, \vec{p})$ is symmetric with respect to $T / 4$, the plot is limited up to $t=T / 4$.

which is indeed defined in such a limit. Using a linear fit in the (bare) quark mass ${ }^{1}$ we get $Z_{V}=0.6679(1)_{\text {stat }}(1)_{\text {syst }}$ at $\beta=2.10$, where the systematic error corresponds to the uncertainty due to different choices of the time extension of the plateau region in Eq. (11).

\footnotetext{
${ }^{1} \mathrm{~A}$ linear dependence on the quark mass is not in contradiction with the $\mathcal{O}(a)$ improvement of the ratio (11), since terms proportional to $a^{2} \mu \Lambda_{\mathrm{QCD}}$ may be dominant with respect to terms proportional to $a^{2} \mu^{2}$.
} 


\section{LATTICE DATA}

\section{A. Choice of time slice sources per gauge configuration}

As mentioned in Sec. III we use stochastic time slice sources for estimating the pion form factor. Therefore, it is interesting to investigate how many time slice sources per gauge configuration are optimal in order to keep the total statistical error still scaling like $1 / \sqrt{N_{\eta}}$ with $N_{\eta}$ being the number of sources per gauge configuration. Due to correlation between time slices one expects that too large values of $N_{\eta}$ do not improve the final error estimate further.

In Fig. 5 we show the relative error of the two-point (left panel) and the three-point (right panel) correlation functions as a function of $N_{\eta}$, at $t / a=24$ for ensemble $\mathrm{cA2}$.09.48. The different source times $t_{0}$ are chosen to be distributed uniformly in the range 0 to $T / a-1$. The solid line represents a fit of the expected ${\sqrt{N_{\eta}}}^{-1}$ behavior to the data.

We observe that the error follows the ${\sqrt{N_{\eta}}}^{-1}$ behavior basically up to $N_{\eta}=12$, where we stopped. Since from $N_{\eta}=8$ on, the error does not improve significantly anymore, we fix $N_{\eta}^{T=96}=12$. This amounts to a mean distance of 12 between source time slices. We keep this mean difference fixed also for the other lattice volumes, i.e., $N_{\eta}^{T=128}=16, N_{\eta}^{T=64}=8$ and $N_{\eta}^{T=48}=4$.

\section{B. Pion electromagnetic form factor}

The lattice data obtained for $F_{\pi}\left(Q^{2}\right)$ as a function of $Q^{2}$ in physical units for some of the gauge ensembles of Table I is shown in Fig. 6. In the left panel data is shown up to $Q^{2}=0.25 \mathrm{GeV}^{2}$, while the right panel restricts $Q^{2}$ to values smaller than $0.12 \mathrm{GeV}^{2}$. In addition to our lattice data we also show experimental data from CERN [3]. The data for all the six gauge ensembles, extracted using the analytic double ratio (20), are collected in the Appendix together with the values chosen for the pion momentum.

It is visible that the errors of our lattice data are compatible with the ones of the experimental data. In particular for small $Q^{2}$ (right panel) the errors of the lattice data are significantly smaller than the errors of the experimental single data points. Of course, the experimental points have a much denser coverage of $Q^{2}$ values. However, thanks to nonperiodic boundary conditions our lattice data covers $Q^{2}$ values below the range where experimental data are available. Moreover, our lattice data at the physical pion point and at the largest volume are compatible with the experimental data within $\approx 2$ standard deviations.

We have collected in Fig. 7 the lattice data for the inverse pion form factor $1 / F_{\pi}$ versus the dimensionless variable $\left(Q / M_{\pi}\right)^{2}$ for the six gauge ensembles of Table I. It can be seen that the data for $1 / F_{\pi}$ exhibit an almost linear behavior with $Q^{2}$, as expected from VMD arguments. Actually the solid lines in Fig. 7 represent the results of a quadratic fit in $Q^{2}$,

$$
\frac{1}{F_{\pi}\left(Q^{2}\right)}=1+s_{\pi}^{\prime}\left(\frac{Q}{M_{\pi}}\right)^{2}+c_{\pi}^{\prime}\left(\frac{Q}{M_{\pi}}\right)^{4}
$$

where we find that $c_{\pi}^{\prime} \ll\left(s_{\pi}^{\prime}\right)^{2}$ in accord with the VMD hypothesis. Moreover, for each pion mass the data for two different lattice volumes are compared in Fig. 7. It can clearly be seen that finite volume effects are relevant for $M_{\pi} L \lesssim 3$.
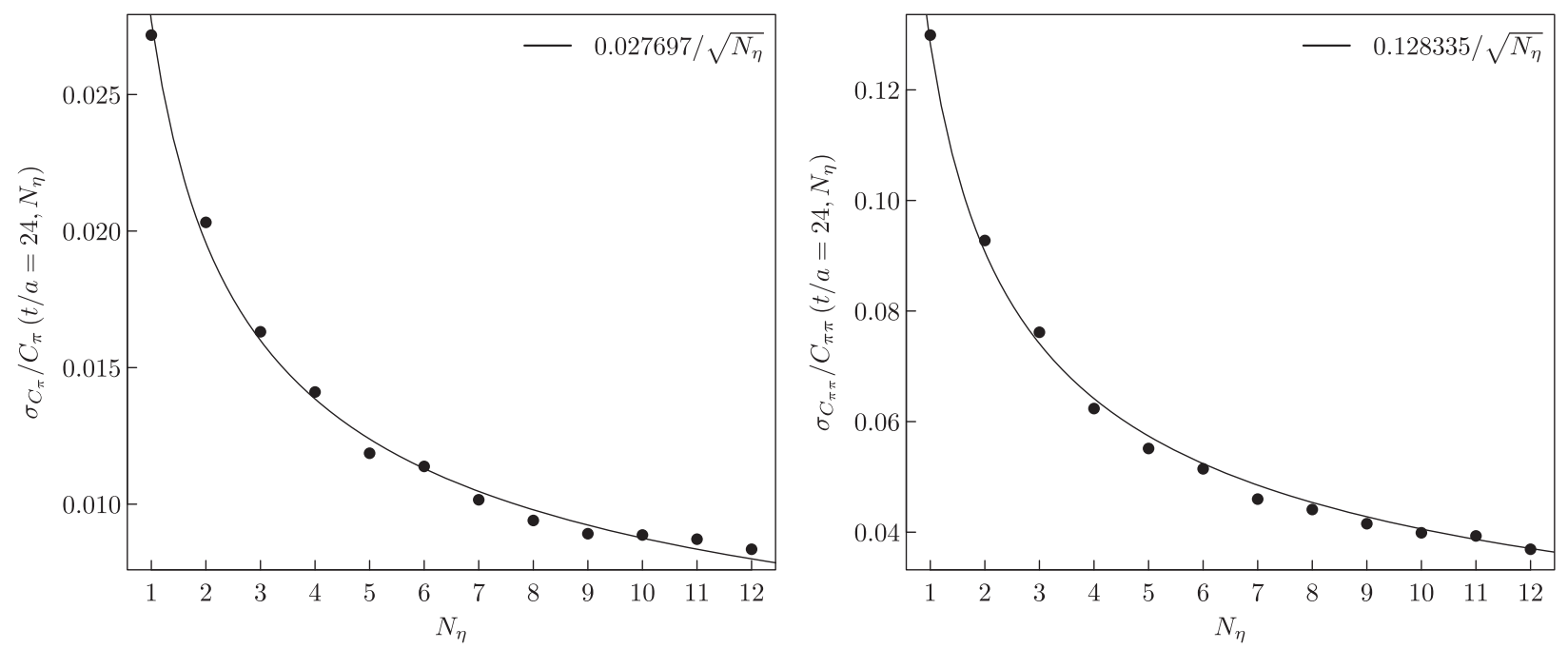

FIG. 5. Relative error in the pion two-point (left) and three-point (right) functions as a functions of the number of sources $N_{\eta}$ per gauge configuration for ensembles cA2.09.48. The solid line represents a fit of $c_{1} / \sqrt{N_{\eta}}$ to the data points. 

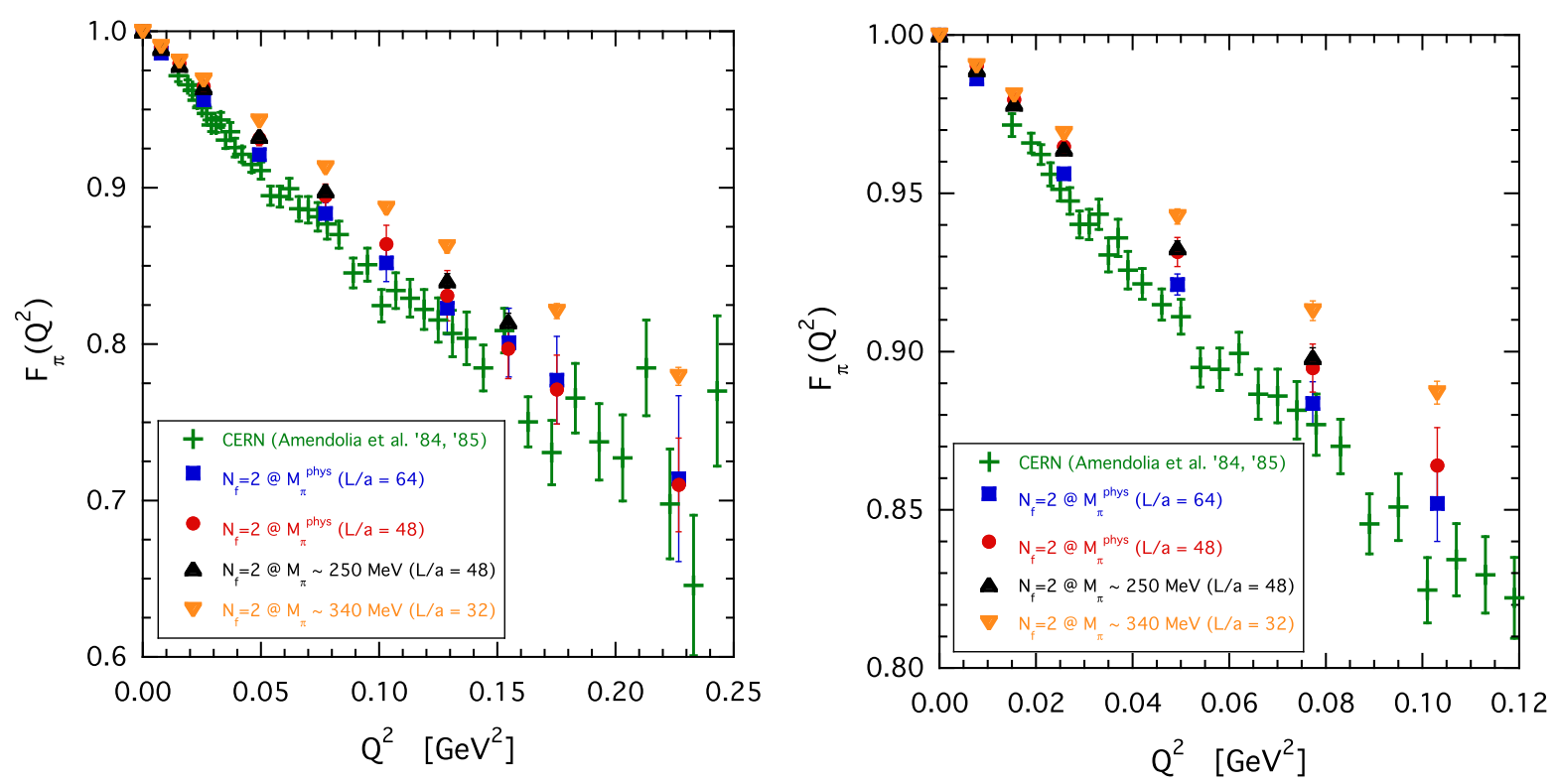

FIG. 6. Data for the vector form factor $F_{\pi}\left(Q^{2}\right)$ as a function of $Q^{2}$ for some of the gauge ensembles used in this work. In addition we show experimental results from CERN [3]. The right panel is a restriction of the left panel to values of $Q^{2}<0.12 \mathrm{GeV}^{2}$.
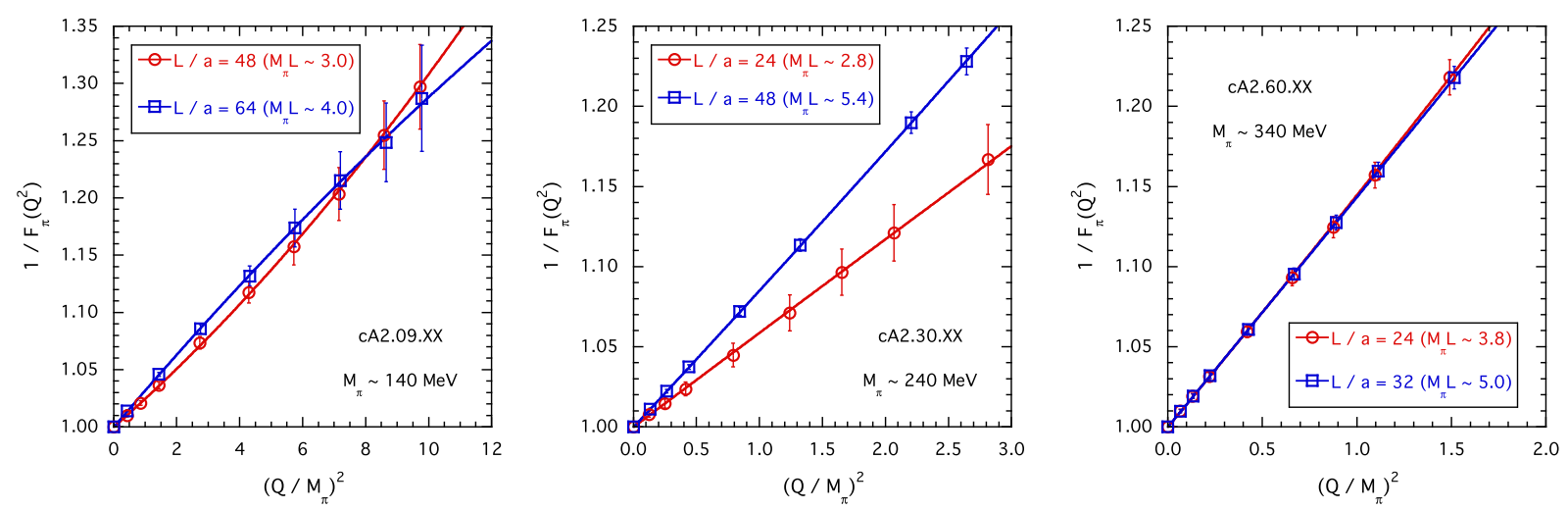

FIG. 7. Data for the inverse pion form factor $1 / F_{\pi}$ as a function of $\left(Q / M_{\pi}\right)^{2}$ for the six ensembles used in this work. In the insets the values of $M_{\pi} L$ are shown. The solid lines represent the results of the quadratic fit (26).

\section{CHIRAL EXTRAPOLATION AND FINITE VOLUME EFFECTS}

\section{A. Chiral extrapolation}

Within SU(2) ChPT the expansion of the pion form factor $F_{\pi}\left(Q^{2}\right)$ in powers of the squared pion mass reads as

$$
F_{\pi}\left(Q^{2}\right)=1+\Delta F_{\pi}^{\mathrm{NLO}}\left(Q^{2}\right)+\Delta F_{\pi}^{\mathrm{NNLO}}\left(Q^{2}\right)+\cdots,
$$

where $\Delta F_{\pi}^{\mathrm{NLO}}\left(Q^{2}\right)$ is the NLO term and $\Delta F_{\pi}^{\mathrm{NNLO}}\left(Q^{2}\right)$ the NNLO one. Both are known [4,5] and the NLO term is explicitly given by

$$
\Delta F_{\pi}^{\mathrm{NLO}}\left(Q^{2}\right)=-\frac{\xi}{3} \frac{Q^{2}}{M_{\pi}^{2}}\left[\bar{\ell}_{6}-\log \frac{\xi}{\xi^{\text {phys }}}-1+R\left(\frac{Q^{2}}{M_{\pi}^{2}}\right)\right]
$$

where $\bar{\ell}_{6}$ is an SU(2) LEC, $\xi \equiv M_{\pi}^{2} /\left(4 \pi f_{\pi}\right)^{2}$ and

$$
R(w)=\frac{2}{3}+\left(1+\frac{4}{w}\right)\left[2+\sqrt{1+\frac{4}{w}} \log \frac{\sqrt{1+\frac{4}{w}}-1}{\sqrt{1+\frac{4}{w}}+1}\right] .
$$

Let us define the slope $s_{\pi}$ and the curvature $c_{\pi}$ of the pion form factor in terms of its expansion in powers of $\left(Q / M_{\pi}\right)^{2}$ as

$$
F_{\pi}\left(Q^{2}\right)=1-s_{\pi} \frac{Q^{2}}{M_{\pi}^{2}}+c_{\pi} \frac{Q^{4}}{M_{\pi}^{4}}+\mathcal{O}\left(\frac{Q^{6}}{M_{\pi}^{6}}\right) .
$$

At NLO one has

$$
s_{\pi}^{\mathrm{NLO}}=\frac{1}{3} \xi\left[\bar{\ell}_{6}-\log \frac{\xi}{\xi^{\mathrm{phys}}}-1\right],
$$




$$
c_{\pi}^{\mathrm{NLO}}=\frac{1}{30} \xi,
$$

which show that the LEC $\bar{\ell}_{6}$ governs only the value of the slope $s_{\pi}^{\text {NLO }}$. Once the value of $\bar{\ell}_{6}$ is fixed by the reproduction of the experimental value of the pion charge radius, i.e., $\bar{\ell}_{6} \simeq 14.6$ (see Ref. [9]), it turns out that $c_{\pi}^{\mathrm{NLO}} \ll\left(s_{\pi}^{\mathrm{NLO}}\right)^{2}$, which is in contradiction with the VMD phenomenology observed both in the experimental data and in our lattice results up to a pion mass of $\simeq 340 \mathrm{MeV}$ (see Fig. 7). In Ref. [9] it was found that, using $\bar{\ell}_{6} \simeq 14.6$ the NLO term (28) works only for very low values of both $Q^{2}\left(Q^{2} \lesssim 0.03 \mathrm{GeV}^{2}\right)$ and the pion mass $\left(M_{\pi} \lesssim 300 \mathrm{MeV}\right)$. Effects from NNLO and higher order terms in the chiral expansion (27) become more and more important as the value of $Q^{2}$ increases. In particular, the curvature $c_{\pi}$ is found to be almost totally dominated by NNLO effects [9]. The latter however depend on several LECs (see Ref. [5]).

In order to avoid the need of many LECs let us consider the inverse of the pion form factor. Using Eq. (27) the $\mathrm{SU}(2) \mathrm{ChPT}$ expansion of $1 / F_{\pi}\left(Q^{2}\right)$ reads as

$$
\begin{aligned}
\frac{1}{F_{\pi}\left(Q^{2}\right)}= & 1-\Delta F_{\pi}^{\mathrm{NLO}}\left(Q^{2}\right)+\left[\left(\Delta F_{\pi}^{\mathrm{NLO}}\left(Q^{2}\right)\right)^{2}\right. \\
& \left.-\Delta F_{\pi}^{\mathrm{NNLO}}\left(Q^{2}\right)\right]+\cdots,
\end{aligned}
$$

where on the rhs the term in the square brackets represent the NNLO correction. Because of the observed VMD phenomenology (see Fig. 7), the NNLO term $\Delta F_{\pi}^{\mathrm{NNLO}}\left(Q^{2}\right)$ in Eq. (33) is expected to be almost compensated by the square of the NLO one $\Delta F_{\pi}^{\mathrm{NLO}}\left(Q^{2}\right)$, leading to a small residual NNLO correction in the inverse pion form factor. This means that $1 / F_{\pi}\left(Q^{2}\right)$ is dominated by the NLO approximation at least in the range of values of $Q^{2}$ and $M_{\pi}$ covered by our simulations, i.e., $Q^{2} \lesssim 0.25 \mathrm{GeV}^{2}$ and $M_{\pi} \lesssim 340 \mathrm{MeV}$. Thus, we can profit from the above feature by using the following ansatz for the chiral extrapolation of the inverse pion form factor

$$
\begin{aligned}
\frac{1}{F_{\pi}\left(Q^{2}\right)}= & 1+\frac{\xi}{3} \frac{Q^{2}}{M_{\pi}^{2}}\left[\bar{\ell}_{6}-\log \frac{\xi}{\xi^{\text {phys }}}-1+R\left(\frac{Q^{2}}{M_{\pi}^{2}}\right)\right] \\
& +\frac{\xi^{2}}{6} \frac{Q^{2}}{M_{\pi}^{2}}\left[b_{1}+b_{2} \frac{Q^{2}}{M_{\pi}^{2}}\right],
\end{aligned}
$$

where the last term in the rhs parametrizes NNLO effects, which we stress are expected to be small. Equation (34) depends only on three unknowns, namely $\bar{\ell}_{6}, b_{1}$ and $b_{2}$, which we determine by fitting our data.

\section{B. Finite volume effects}

As illustrated in Fig. 7, our data for the pion form factor suffer from FVEs. In this work we follow three strategies to correct for FVEs, profiting from the two lattice volumes available at each value of the quark mass.
We first introduce the FVE factor $K_{\mathrm{FVE}}\left(Q^{2}, L\right)$ defined as

$$
F_{\pi}\left(Q^{2}, L\right)=F_{\pi}\left(Q^{2}, \infty\right)+K_{\mathrm{FVE}}\left(Q^{2}, L\right),
$$

which implies

$\frac{1}{F_{\pi}\left(Q^{2}, L\right)}=\frac{1}{F_{\pi}\left(Q^{2}, \infty\right)}\left[1-\frac{1}{F_{\pi}\left(Q^{2}, \infty\right)} K_{\mathrm{FVE}}\left(Q^{2}, L\right)\right]$,

where $1 / F_{\pi}\left(Q^{2}, \infty\right)$ is given by Eq. (34).

The three strategies are as follows:

(i) Make use of the $\mathrm{SU}(2) \mathrm{ChPT}$ prediction derived at NLO in the Breit frame $[33,34]$. The correction factor $K_{\mathrm{FVE}}\left(Q^{2}, L\right)$ reads explicitly

$$
\begin{aligned}
K_{\mathrm{FVE}}\left(Q^{2}, L\right)= & \frac{C}{f_{\pi}^{2}}\left\{\int _ { 0 } ^ { 1 } d x I _ { 1 / 2 } \left[(1-2 x) \frac{2 \pi \vec{\theta}}{L} ;\right.\right. \\
& \left.M_{\pi}^{2}+x(1-x) Q^{2}\right] \\
& \left.-I_{1 / 2}\left[\frac{2 \pi \vec{\theta}}{L} ; M_{\pi}^{2}\right]\right\},
\end{aligned}
$$

where $C$ is a parameter to be determined in the fitting procedure, $Q^{2}=4(2 \pi \vec{\theta} / L)^{2}$ and

$$
\begin{aligned}
I_{1 / 2}\left[\frac{2 \pi \vec{\theta}}{L} ; M_{\pi}^{2}\right]= & \frac{1}{2 \pi^{3 / 2} L^{2}} \int_{0}^{\infty} d \tau \frac{1}{\sqrt{\tau}} e^{-\tau\left(\frac{M_{\pi} L}{2 \pi}\right)^{2}} \\
& \times\left[\prod_{i=1}^{3} \Theta\left(\tau, \theta_{i}\right)-\left(\frac{\pi}{\tau}\right)^{3 / 2}\right]
\end{aligned}
$$

with $\Theta(\tau, \theta) \equiv \sum_{n=-\infty}^{\infty} e^{-\tau(n+\theta)^{2}}$ being the elliptic Jacobi function.

(ii) Use a phenomenological ansatz, inspired by the asymptotic expansion of Eq. (37), given by

$$
K_{\mathrm{FVE}}\left(Q^{2}, L\right)=\frac{Q^{2}}{M_{\pi}^{2}}\left[C_{1}+C_{2} \frac{Q^{2}}{M_{\pi}^{2}}\right] \frac{\xi}{\left(M_{\pi} L\right)^{3 / 2}} \cdot e^{-M_{\pi} L},
$$

where $C_{1}$ and $C_{2}$ are parameters to be determined in the fitting procedure.

(iii) Use only the largest volume available at each pion mass and assume that FVEs are negligible for these volumes (i.e., putting $K_{\mathrm{FVE}}=0$ ).

We want to point out that our fitting ansatz (36) is defined in terms of dimensionless quantities only, namely $\xi$, $Q^{2} / M_{\pi}^{2}$ and $M_{\pi} L$, and therefore the knowledge of the lattice scale is not required. 

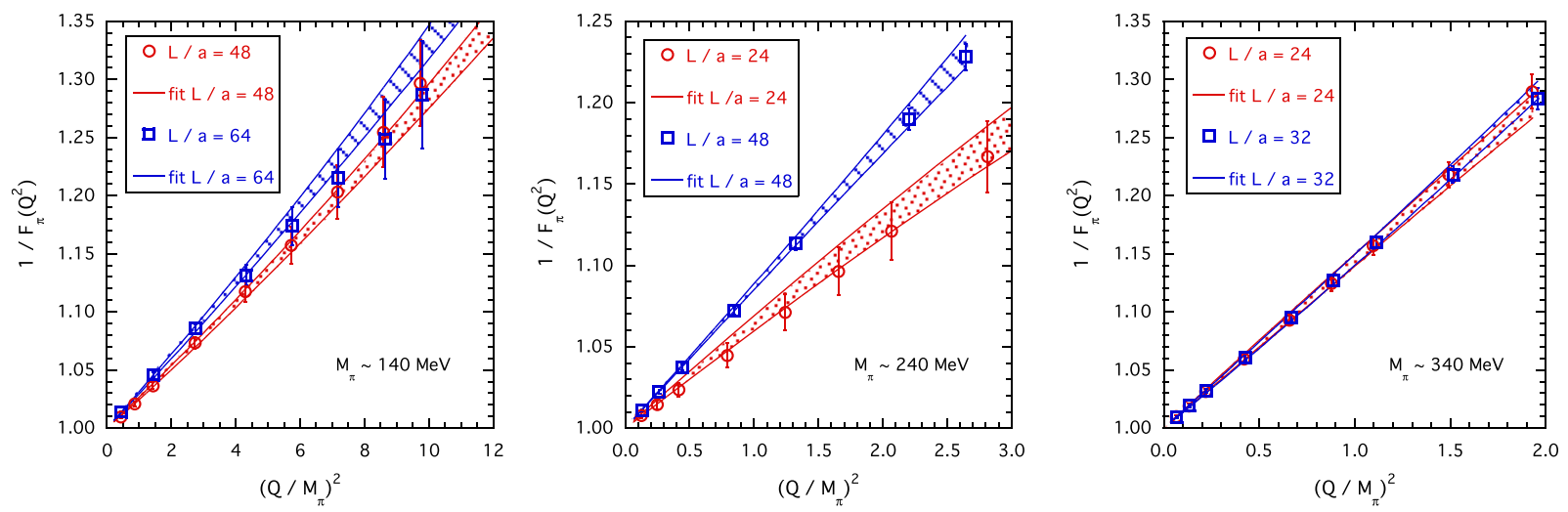

FIG. 8. Results of our fitting procedure of the inverse pion form factor, based on Eq. (36) with $1 / F_{\pi}\left(Q^{2}, \infty\right)$ and $K_{\mathrm{FVE}}\left(Q^{2}, L\right)$ given by Eqs. (34) and (37), respectively, for all the six ensembles of Table I. The bands correspond to statistical uncertainties only.

\section{EXTRAPOLATIONS TO THE PHYSICAL POINT}

In this section we perform the chiral and infinite volume extrapolations of the lattice data adopting our fitting ansatz (36). Various sources of systematic effects have been taken into account, namely

(i) The numerical and analytical estimates of the pion form factor given by Eqs. (18) and (21), respectively. The corresponding uncertainty will be denoted by ()$_{\text {ratio }}$;

(ii) The time extensions $\left[t_{\min }, T / 2-t_{\min }\right]$ chosen for the plateaux of the double ratios (17) and (20) corresponding to $t_{\min }=10$ and 12 . The corresponding uncertainty will be denoted by ()$_{\text {fit-range }}$;

(iii) Either the inclusion of all the six gauge ensembles of Table I or the restriction to the two gauge ensembles cA2.09.XX at the physical pion mass. The corresponding uncertainty will be denoted by ()$_{M_{\pi}}$;

(iv) Either the inclusion $\left(b_{1} \neq 0\right.$ and $\left.b_{2} \neq 0\right)$ or the exclusion $\left(b_{1}=b_{2}=0\right)$ of the NNLO effects in Eq. (34). The corresponding uncertainty will be denoted by ()$_{\mathrm{ChPT}}$;

(v) The FVEs evaluated according to the three procedures $\mathrm{A}, \mathrm{B}$ and $\mathrm{C}$, described in Sec. VB. The corresponding uncertainty will be denoted by ()$_{\mathrm{FVE}}$;

(vi) The inclusion of all $Q^{2}$ values or the restriction to $Q^{2} \leq 2 M_{\pi}^{2}$. The corresponding uncertainty will be denoted by ()$_{Q^{2} \text {-range. }}$

The quality of our fitting procedure is illustrated in Fig. 8 for all the six ensembles used in this work. ${ }^{2}$ It can be seen that within the statistical errors the FVEs present in the data are nicely reproduced both at small and large values of the pion momentum by the ChPT ansatz given by Eq. (37).

\footnotetext{
${ }^{2}$ The values of the fitting parameters are determined by a $\chi^{2}-$ minimization procedure adopting an uncorrelated $\chi^{2}$. The resulting values of $\chi^{2} /$ d.o.f. do not exceed $\simeq 1$.3. The results of the various fits are averaged according to Eq. (28) of Ref. [35].
}

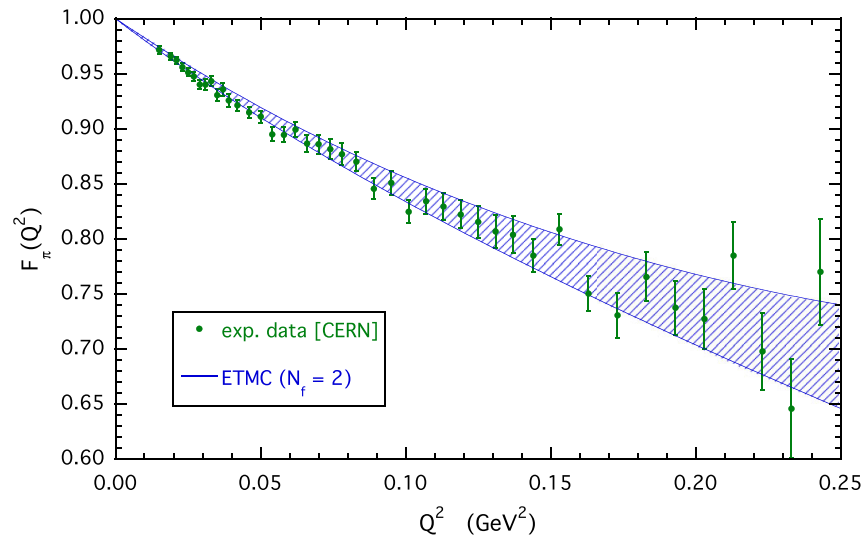

FIG. 9. Comparison of the $N_{f}=2$ ETMC values for $F_{\pi}\left(Q^{2}\right)$, extrapolated to the physical pion point and to infinite volume limit, with the experimental data from CERN [3]. The band includes both statistical and systematic errors.

Similar results hold as well also in the case of the phenomenological fitting function (39).

The results for the pion form factor, extrapolated at the physical pion point and in the infinite volume limit, are compared with the experimental data from CERN [3] in Fig. 9.

As far as the pion charge radius is concerned, our fitting ansatz (36) implies that at the physical pion point and the infinite volume limit one has

$$
\left\langle r^{2}\right\rangle_{\pi}=\frac{1}{\left(4 \pi f_{\pi}\right)^{2}}\left[2\left(\bar{e}_{6}-1\right)+\frac{M_{\pi}^{2}}{\left(4 \pi f_{\pi}\right)^{2}} b_{1}\right] .
$$

Thus, our final result at a fixed lattice spacing $(a \simeq 0.09 \mathrm{fm})$ reads

$$
\begin{aligned}
\left\langle r^{2}\right\rangle_{\pi}= & 0.443(21)_{\text {stat }}(7)_{\text {ratio }}(1)_{\text {fit-range }}(7)_{M_{\pi}}(6)_{\mathrm{ChPT}} \\
& \times(15)_{\mathrm{FVE}}(6)_{Q^{2}-\text { range }} \mathrm{fm}^{2} \\
= & 0.443(21)_{\text {stat }}(20)_{\text {syst }} \mathrm{fm}^{2} \\
= & 0.443(29) \mathrm{fm}^{2},
\end{aligned}
$$


which is consistent with the experimental value $\left\langle r^{2}\right\rangle_{\pi}^{\exp }=$ $0.452(11) \mathrm{fm}^{2}$ from PDG [19] as well as with the recent high-precision determination $\left\langle r^{2}\right\rangle_{\pi}=0.4320(41) \mathrm{fm}^{2}$ [36], obtained using experimental data on the pion form factor from both timelike and spacelike regions. This suggests that the impact of discretization effects on our result (1) could be small with respect to the other sources of uncertainties.

The lattice calculations of $\left\langle r^{2}\right\rangle_{\pi}$ have been analyzed recently by FLAG and are collected in Table 22 of Ref. [37]. Four results satisfy the FLAG quality criteria, namely, $\left\langle r^{2}\right\rangle_{\pi}=0.441(66) \mathrm{fm}^{2} \quad$ [8] $\quad\left(N_{f}=2\right),\left\langle r^{2}\right\rangle_{\pi}=$ $0.456(38) \mathrm{fm}^{2}$ [9] $\left(N_{f}=2\right),\left\langle r^{2}\right\rangle_{\pi}=0.481(35) \mathrm{fm}^{2}$ [13] $\left(N_{f}=2\right)$ and $\left\langle r^{2}\right\rangle_{\pi}=0.403(19) \mathrm{fm}^{2} \quad$ [16] $\quad\left(N_{f}=2+\right.$ $1+1)$. Our finding (41) is nicely consistent with all the above lattice results.

The value of the NLO SU(2) LEC $\bar{\ell}_{6}$, appearing in Eq. (40) and corresponding to our result (41), is equal to

$$
\begin{aligned}
\bar{\ell}_{6}= & 16.21(76)_{\text {stat }}(25)_{\text {ratio }}(3)_{\text {fit-range }}(26)_{M_{\pi}}(24)_{\mathrm{ChPT}} \\
& \times(50)_{\mathrm{FVE}}(20)_{Q^{2} \text {-range }} \\
= & 16.21(76)_{\text {stat }}(70)_{\text {syst }} \\
= & 16.21(1.03) .
\end{aligned}
$$

\section{SUMMARY AND DISCUSSION}

We have presented an investigation of the electromagnetic pion form factor, $F_{\pi}\left(Q^{2}\right)$, at small values of the fourmomentum transfer $Q^{2}\left(\lesssim 0.25 \mathrm{GeV}^{2}\right)$, based on the gauge configurations generated by ETMC with $N_{f}=2$ twistedmass quarks at maximal twist including a clover term. Momentum is injected using nonperiodic boundary conditions and the calculations are carried out at a fixed lattice spacing $(a \simeq 0.09 \mathrm{fm})$ and with pion masses equal to its physical value, $240 \mathrm{MeV}$ and $340 \mathrm{MeV}$. We have successfully analyzed our data using chiral perturbation theory at next-toleading order in the light-quark mass. For each pion mass two different lattice volumes are used to take care of finite size effects. Our final result for the squared charge radius is $\left\langle r^{2}\right\rangle_{\pi}=0.443(29) \mathrm{fm}^{2}$, where the error includes several sources of systematic errors except the uncertainty related to discretization effects. The corresponding value of the SU(2) low-energy constant $\bar{\ell}_{6}$ is equal to $\bar{\ell}_{6}=16.2(1.0)$. Our result is consistent with the experimental value $\left\langle r^{2}\right\rangle_{\pi}^{\exp }=$ $0.452(11) \mathrm{fm}^{2}$ [19] as well as with other lattice estimates (see Ref. [37]). This suggests that the impact of discretization effects on our result could be small with respect to the other sources of uncertainties.

\section{ACKNOWLEDGMENTS}

We thank the members of ETMC for the most enjoyable collaboration. The computer time for this project was made available to us by the John von Neumann-Institute for Computing (NIC) on the Jureca and Juqueen systems in Jülich, and by the Gauss Centre for Supercomputing under Project No. PR74YO on the GCS Supercomputer SuperMUC at Leibniz Supercomputing Centre. This work was granted access also to the HPC resources of CINES and IDRIS under the allocation 52271 made by Grand equipment national de calcul intensif. This project was funded by the Deutsche Forschungsgemeinschaft as a project in the Sino-German CRC110 and by the Horizon 2020 research and innovation program of the European Commission under the Marie Sklodowska-Curie Grant Agreement No. 642069. S. B. is supported by the latter program. The open source software packages tmLQCD [38-40], Lemon [41], $\mathrm{DD} \alpha \mathrm{AMG}$ [42] and R [43] have been used.

\section{APPENDIX: PION MOMENTA AND FORM FACTOR DATA}

In Tables_II-VII we collect the values adopted for the vector $\vec{\theta}=\{\theta, \theta, \theta\}$, the squared three-momentum $|\vec{p}|^{2}=4 \pi^{2}|\vec{\theta}|^{2} / L^{2}$ in lattice units, the squared fourmomentum transfer $Q^{2}=-q^{2}=4|\vec{p}|^{2}$ in units of the pion mass and the values of the pion form factor $F_{\pi}\left(Q^{2}\right)$, extracted using the analytic double ratio (20), in the case of the six ensembles of Table I.

TABLE II. Values of the angle $\theta$, the squared three-momentum $|\vec{p}|^{2}$ in lattice units, the squared four-momentum transfer $Q^{2}$ in units of the pion mass and the values of the pion form factor $F_{\pi}\left(Q^{2}\right)$ for the ensemble cA2.09.48. Errors are statistical only.

\begin{tabular}{lccl}
\hline \hline$\theta$ & $|a \vec{p}|^{2}$ & $Q^{2} / M_{\pi}^{2}$ & \multicolumn{1}{c}{$F_{\pi}\left(Q^{2}\right)$} \\
\hline 0 & 0 & 0 & $1.000000(0)$ \\
0.0898 & 0.000414525 & 0.42912 & $0.9903(12)$ \\
0.1270 & 0.000829098 & 0.85828 & $0.9796(20)$ \\
0.16395 & 0.00138172 & 1.4312 & $0.9648(28)$ \\
0.2268 & 0.00264414 & 2.7372 & $0.9315(46)$ \\
0.2840 & 0.00414606 & 4.2920 & $0.8948(76)$ \\
0.32795 & 0.00552858 & 5.7249 & $0.864(12)$ \\
0.36665 & 0.00691038 & 7.1517 & $0.831(16)$ \\
0.40165 & 0.00829266 & 8.5867 & $0.797(19)$ \\
0.4276 & 0.00939883 & 9.7297 & $0.771(22)$ \\
0.4864 & 0.0121615 & 12.590 & $0.710(30)$ \\
\hline \hline
\end{tabular}

TABLE III. The same as in Table II but for the ensemble cA2.09.64.

\begin{tabular}{lccl}
\hline \hline$\theta$ & $|a \vec{p}|^{2}$ & $Q^{2} / M_{\pi}^{2}$ & \multicolumn{1}{c}{$F_{\pi}\left(Q^{2}\right)$} \\
\hline 0 & 0 & 0 & $1.000000(0)$ \\
0.11975 & 0.000414641 & 0.43059 & $0.98616(68)$ \\
0.2186 & 0.00138172 & 1.4361 & $0.9562(16)$ \\
0.3024 & 0.00264414 & 2.7482 & $0.9212(33)$ \\
0.37865 & 0.00414569 & 4.3076 & $0.8836(69)$ \\
0.43725 & 0.00552816 & 5.7470 & $0.852(12)$ \\
0.48885 & 0.00690991 & 7.1832 & $0.823(17)$ \\
0.5360 & 0.00830712 & 8.6339 & $0.801(22)$ \\
0.5701 & 0.00939773 & 9.7675 & $0.777(28)$ \\
0.64855 & 0.0121621 & 12.639 & $0.714(53)$ \\
\hline \hline
\end{tabular}


TABLE IV. The same as in Table II but for the ensemble cA2.30.24.

\begin{tabular}{lccl}
\hline \hline$\theta$ & \multicolumn{1}{c}{$|a \vec{p}|^{2}$} & $Q^{2} / M_{\pi}^{2}$ & \multicolumn{1}{c}{$F_{\pi}\left(Q^{2}\right)$} \\
\hline 0 & 0 & 0 & $1.000000(0)$ \\
0.0449 & 0.000414525 & 0.12394 & $0.9922(18)$ \\
0.0635 & 0.000829098 & 0.24788 & $0.9856(28)$ \\
0.0820 & 0.00138257 & 0.41336 & $0.9769(41)$ \\
0.1134 & 0.00264414 & 0.79054 & $0.9571(68)$ \\
0.1420 & 0.00414606 & 1.2396 & $0.9336(97)$ \\
0.16395 & 0.0055269 & 1.6534 & $0.912(12)$ \\
0.1833 & 0.00690849 & 2.0655 & $0.892(14)$ \\
0.2138 & 0.00939883 & 2.8101 & $0.857(16)$ \\
0.2432 & 0.0121615 & 3.6360 & $0.823(19)$ \\
0.27835 & 0.0159309 & 4.7613 & $0.781(22)$ \\
0.3154 & 0.0204542 & 6.1154 & $0.734(26)$ \\
0.3608 & 0.0267665 & 8.0026 & $0.681(36)$ \\
0.40205 & 0.0332368 & 9.9396 & $0.647(58)$ \\
0.4536 & 0.0423063 & 12.649 & $0.61(10)$ \\
\hline \hline
\end{tabular}

TABLE V. The same as in Table II but for the ensemble cA2.30.48.

\begin{tabular}{lccl}
\hline \hline$\theta$ & $|a \vec{p}|^{2}$ & $Q^{2} / M_{\pi}^{2}$ & \multicolumn{1}{c}{$F_{\pi}\left(Q^{2}\right)$} \\
\hline 0 & 0 & 0 & $1.000000(0)$ \\
0.0898 & 0.000414525 & 0.13224 & $0.98908(68)$ \\
0.1270 & 0.000829098 & 0.26449 & $0.9782(10)$ \\
0.16395 & 0.00138172 & 0.44105 & $0.9640(14)$ \\
0.2268 & 0.00264414 & 0.84350 & $0.9328(22)$ \\
0.2840 & 0.00414606 & 1.3226 & $0.8981(31)$ \\
0.36665 & 0.00691038 & 2.2039 & $0.8405(47)$ \\
0.40165 & 0.00829266 & 2.6461 & $0.8143(55)$ \\
\hline \hline
\end{tabular}

TABLE VI. The same as in Table II but for the ensemble $\mathrm{cA} 2.60 .24$.

\begin{tabular}{llll}
\hline \hline$\theta$ & \multicolumn{1}{c}{$|a \vec{p}|^{2}$} & $Q^{2} / M_{\pi}^{2}$ & \multicolumn{1}{c}{$F_{\pi}\left(Q^{2}\right)$} \\
\hline 0 & 0 & 0 & $1.000000(0)$ \\
0.0449 & 0.000414525 & 0.065909 & $0.9901(11)$ \\
0.0635 & 0.000829098 & 0.13183 & $0.9811(16)$ \\
0.0820 & 0.00138257 & 0.21983 & $0.9694(22)$ \\
0.1134 & 0.00264414 & 0.42042 & $0.9439(33)$ \\
0.1420 & 0.00414606 & 0.65922 & $0.9148(43)$ \\
0.16395 & 0.0055269 & 0.87931 & $0.8892(52)$ \\
0.1833 & 0.00690849 & 1.0984 & $0.8642(60)$ \\
0.2138 & 0.00939883 & 1.4944 & $0.8210(74)$ \\
0.2432 & 0.0121615 & 1.9337 & $0.7754(90)$ \\
\hline \hline
\end{tabular}

TABLE VII. The same as in Table II but for the ensemble cA2.60.32.

\begin{tabular}{llll}
\hline \hline$\theta$ & \multicolumn{1}{c}{$|a \vec{p}|^{2}$} & $Q^{2} / M_{\pi}^{2}$ & \multicolumn{1}{c}{$F_{\pi}\left(Q^{2}\right)$} \\
\hline 0 & 0 & 0 & $1.000000(0)$ \\
0.05985 & 0.000414295 & 0.066720 & $0.99035(95)$ \\
0.08465 & 0.000828772 & 0.13340 & $0.9811(13)$ \\
0.10935 & 0.00138299 & 0.22255 & $0.9690(17)$ \\
0.1512 & 0.00264414 & 0.42511 & $0.9427(24)$ \\
0.18935 & 0.00414679 & 0.66635 & $0.9129(31)$ \\
0.2186 & 0.0055269 & 0.88859 & $0.8870(36)$ \\
0.2444 & 0.00690849 & 1.1107 & $0.8624(41)$ \\
0.28505 & 0.00939773 & 1.5115 & $0.8211(48)$ \\
0.32425 & 0.0121602 & 1.9545 & $0.7794(57)$ \\
\hline \hline
\end{tabular}

[1] H. P. Blok et al. (Jefferson Lab Collaboration), Phys. Rev. C 78, 045202 (2008).

[2] G. M. Huber et al. (Jefferson Lab Collaboration), Phys. Rev. C 78, 045203 (2008).

[3] S. R. Amendolia et al. (NA7 Collaboration), Nucl. Phys. B277, 168 (1986).

[4] J. Gasser and H. Leutwyler, Ann. Phys. (N.Y.) 158, 142 (1984).

[5] J. Bijnens, G. Colangelo, and P. Talavera, J. High Energy Phys. 05 (1998) 014.

[6] G. Martinelli and C. T. Sachrajda, Nucl. Phys. B306, 865 (1988).

[7] T. Draper, R. M. Woloshyn, W. Wilcox, and K.-F. Liu, Nucl. Phys. B318, 319 (1989).

[8] D. Brommel et al. (QCDSF/UKQCD Collaboration), Eur. Phys. J. C 51, 335 (2007).
[9] R. Frezzotti, V. Lubicz, and S. Simula (ETM Collaboration), Phys. Rev. D 79, 074506 (2009).

[10] P. A. Boyle et al., J. High Energy Phys. 07 (2008) 112.

[11] S. Aoki et al. (TWQCD and JLQCD Collaborations), Phys. Rev. D 80, 034508 (2009).

[12] O. H. Nguyen, K.-I. Ishikawa, A. Ukawa, and N. Ukita, J. High Energy Phys. 04 (2011) 122.

[13] B. B. Brandt, A. Jüttner, and H. Wittig, J. High Energy Phys. 11 (2013) 034.

[14] H. Fukaya, S. Aoki, S. Hashimoto, T. Kaneko, H. Matsufuru, and J. Noaki, Phys. Rev. D 90, 034506 (2014).

[15] S. Aoki, G. Cossu, X. Feng, S. Hashimoto, T. Kaneko, J. Noaki, and T. Onogi (JLQCD Collaboration), Phys. Rev. D 93, 034504 (2016).

[16] J. Koponen, F. Bursa, C. T. H. Davies, R. J. Dowdall, and G. P. Lepage, Phys. Rev. D 93, 054503 (2016). 
[17] A. Abdel-Rehim et al. (ETM Collaboration), Phys. Rev. D 95, 094515 (2017).

[18] R. Frezzotti and G. C. Rossi, J. High Energy Phys. 08 (2004) 007.

[19] C. Patrignani et al. (Particle Data Group Collaboration), Chin. Phys. C 40, 100001 (2016).

[20] R. Frezzotti, P. A. Grassi, S. Sint, and P. Weisz (ALPHA Collaboration), J. High Energy Phys. 08 (2001) 058.

[21] Y. Iwasaki, Nucl. Phys. B258, 141 (1985).

[22] B. Sheikholeslami and R. Wohlert, Nucl. Phys. B259, 572 (1985).

[23] T. Chiarappa, F. Farchioni, K. Jansen, I. Montvay, E. E. Scholz, L. Scorzato, T. Sudmann, and C. Urbach, Eur. Phys. J. C 50, 373 (2007).

[24] R. Baron et al. (ETM Collaboration), J. High Energy Phys. 06 (2010) 111.

[25] P. F. Bedaque, Phys. Lett. B 593, 82 (2004).

[26] G. M. de Divitiis, R. Petronzio, and N. Tantalo, Phys. Lett. B 595, 408 (2004).

[27] D. Guadagnoli, F. Mescia, and S. Simula, Phys. Rev. D 73, 114504 (2006).

[28] C. T. Sachrajda and G. Villadoro, Phys. Lett. B 609, 73 (2005).

[29] P. F. Bedaque and J. W. Chen, Phys. Lett. B 616, 208 (2005).

[30] J. M. Flynn, A. Juttner, and C. T. Sachrajda (UKQCD Collaboration), Phys. Lett. B 632, 313 (2006).
[31] R. Baron et al. (ETM Collaboration), Proc. Sci., LAT2007 (2007) 153 [arXiv:0710.1580].

[32] F. J. Jiang and B. C. Tiburzi, Phys. Lett. B 645, 314 (2007).

[33] F.-J. Jiang and B.C. Tiburzi, Phys. Rev. D 78, 037501 (2008).

[34] G. Colangelo and A. Vaghi, J. High Energy Phys. 07 (2016) 134.

[35] N. Carrasco et al. (ETM Collaboration), Nucl. Phys. B887, 19 (2014).

[36] B. Ananthanarayan, I. Caprini, and D. Das, Phys. Rev. Lett. 119, 132002 (2017).

[37] S. Aoki et al., Eur. Phys. J. C 77, 112 (2017).

[38] K. Jansen and C. Urbach, Comput. Phys. Commun. 180, 2717 (2009).

[39] A. Deuzeman, K. Jansen, B. Kostrzewa, and C. Urbach, Proc. Sci., LATTICE2013 (2013) 416 [arXiv:1311.4521].

[40] A. Abdel-Rehim et al., Proc. Sci., LATTICE2013 (2014) 414 [arXiv:1311.5495].

[41] A. Deuzeman, S. Reker, and C. Urbach (ETM Collaboration), Comput. Phys. Commun. 183, 1321 (2012).

[42] C. Alexandrou, S. Bacchio, J. Finkenrath, A. Frommer, K. Kahl, and M. Rottmann, Phys. Rev. D 94, 114509 (2016).

[43] R Development Core Team Collaboration, R: A Language and Environment for Statistical Computing (R Foundation for Statistical Computing, Vienna, Austria, 2005). 